TRABAJOS DE PREHISTORIA

55, n. $^{\circ} 1,1998$, pp. 147 a 154

\section{INVESTIGACIÓN \\ ARQUEOLÓGICA EN LAS ESTEPAS DE EURASIA}

\author{
ARCHAEOLOGICAL RESEARCH \\ IN THE EURASIAN STEPPES
}

HERMANN PARZINGER (*)

\section{RESUMEN}

Este artículo presenta las actividades de la 'EurasienAbteilung', del Instituto Arqueológico Alemán, en los distintos países de la ex-URSS. Como muchos de estos proyectos han empezado hace poco tiempo, no pretendemos adelantar resultados, sino planteamientos científicos. El área que abarcan se extiende desde el Mar Negro hasta el Noreste de China. Sus principales temas de investigación son: el cambio del Bronce Final a la primera Edad del Hierro en el norte del Mar Negro, la colonización griega en esta zona, las actividades de los Escitas y de los Sasánidas en Transcaucasia, el urbanismo y la metalurgia de la Edad del Bronce en Asia Central y, finalmente, el desarrollo cultural desde el Bronce Antiguo hasta la época de los Escitas y Hunos en el sur de Siberia.

\section{ABSTRACT}

This paper presents the activities of the 'Eurasien-Abteilung' of the German Archaeological Institute in different countries of former USSR. Many of these projects have just begun; consequently the paper does not discuss the results of these investigations, but details their scientific purposes. The investigations cover an area which extends from the Black Sea to northeastern China. The principal objects of these investigations include: the transition from the Late Bronze to the Early Iron Age in the Pontic area north of the Black Sea and the Greek colonization of that area; the activities of the Scythians and the Sassanians in Transcaucasia, urbanism and metallurgy in the Bronze Age of Central Asia; and, finally, cul-

(*) Deutsches Archäologisches Institut, Eurasien-Abteilung, Im Dol 2-6, D-14195 Berlin, Tel.: 030/83008-311, Fax: 030/ 83008-313, e-mail: eurasien@zedat.fu-berlin.de.

El artículo fue remitido en su versión final el 5-XII-97. tural developments from the Early Bronze Age to the periods of the Scythians and the Huns period in southern Siberia.

Palabras clave: Eurasia. Mar Negro. Caúcaso. Asia Central. Siberia. Estepas. Edad del Bronce. Colonización griega. Estilo animalístico. Metalurgia. Kurgán. Escitas. Hunos. Sasánidas.

Key words: Eurasian. Black Sea. Caucasus. Central Asia. Siberia. Steppes. Bronze Age. Greek Colonization. Animal Style. Metallurgy. Kurgan. Scythians. Huns. Sassanians.

El Instituto Arqueológico Alemán (Deutsches Archäologisches Institut) ha fundado en el año 1995 un nuevo departamento, la 'Eurasien-Abteilung', con sede en Berlin, para ampliar el campo de investigación arqueológica a las estepas euroasiáticas y las civilizaciones al Sur de ellas (Parzinger, 1995). El territorio del cual se ocupa la 'Eurasien-Abteilung', incluye la zona del Mar Negro (Moldavia, Ucrania, Rusia del Sur), el Caúcaso (las repúblicas autónomas de la Federación Rusa en el Norte del Caúcaso, Georgia, Azerbaiyán y Armenia), Asia Central (Irán, Turkmenistán, Uzbekistán, Kazajstán, Kirgistán, Tachikistán, Afganistán y Pakistán) y Siberia, Tuva, Mongolia y el Norte de China. En la actualidad existen ya varios convenios de cooperación con distintas instituciones en Rusia (Moscú, St. Petersburgo, Minusinsk), Ucrania, Georgia, Uzbekistán, Tachikistán, Kazajstán y las repúblicas autónomas de la Federación Rusa de Dagestán, Jakassia y Tuva. Además están en curso otros convenios con los Institutos Arqueológicos de Pekin y Hohot, este 
último en la provincia de Mongolia Interior en el Norte de China (Parzinger, 1995).

La cooperación con estos países no se concentra solamente en excavaciones comúnes, sino también en el intercambio de especialistas a través de becas, intercambio bibliográfico y proyectos de publicación. La 'Eurasien-Abteilung', que incluye ahora también la antigua 'Abteilung Teheran' del Instituto Arqueológico Alemán, edita dos revistas (Eurasia Antiqua e Archäologische Mitteilungen aus Iran und Turan) y seis series de monografías (Archäologie in Eurasien, Archäologie in Iran und Turan, Materialien zur Iranischen Archäologie, Iranische Denkmäler, Steppenvölker Eurasiens y Pontus Septentrionalis). Las dos últimas, bilingües, se editan junto con el Instituto de Historia de la Academia de Ciencias de Rusia con textos en alemán y ruso. Estas revistas y series están siendo ya utilizadas con relativa frecuencia por colegas de la CEI, que presentan en ellas sus materiales e ideas a un público de carácter internacional.

Los proyectos de investigación de la 'EurasienAbteilung' se extienden del Mar Negro hasta el Noreste de China (Fig. 1) y abarcan problemas de distintos períodos. Se pueden distinguir excavaciones, prospecciones y estudios de materiales. Se realizan siempre en colaboración con los institutos arqueológicos de los distintos países.

Empezando en el Oeste de las estepas euroasiáticas, queremos presentar en primer lugar los proyectos en la zona del Mar Negro. En cooperación con la Universidad de Estambul se investiga allí un 'tell' neolítico (Asagi Pinar) y uno de la primera Edad del Bronce (Kanligecit), al sur de la capital provincial de la Tracia turca, Kirklareli. Estos trabajos son sumamente importantes para todas aquellas cuestiones relacionadas con los contactos entre Anatolia, los Balcanes y las regiones al Noroeste del Mar Negro. En Asagi Pinar está representado casi todo el desarrollo del Neolítico desde el antiguo (Karanovo I) hasta el Neolítico reciente (Karanovo IV) y las extensas áreas de excavación no solamente dan abundantes materiales -tanto líticos como cerámicos- para definir los distintas etapas de este desarrollo, sino que también permiten entender mejor la arquitectura y la organización de los distintos poblados superpuestos. Pese al abandono definitivo del 'tell' de Asagi Pinar por razones hasta ahora desconocidas durante el Neo-

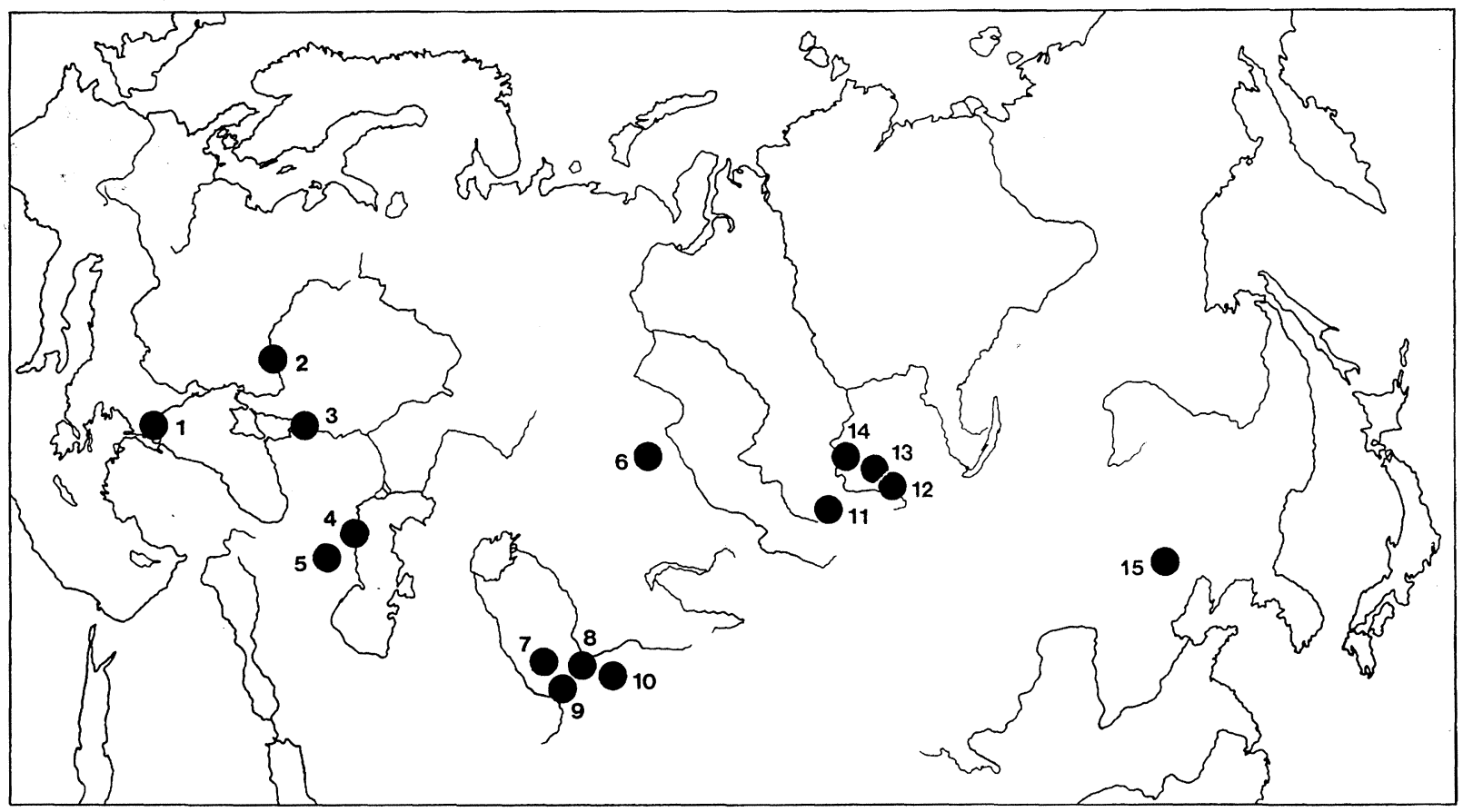

Fig. 1. Mapa de los proyectos de investigación de la Eurasien-Abteilung. 1. Kirklareli; 2. Subotiv; 3. Tanais; 4. Derbent; 5. Ciskaraant-Gora; 6. Sergeevka; 7. Karnab; 8. Mushiston; 9. Dzharkutan-Tepe; 10. Kyzyl-Su; 11. Tuva occidental; 12. Doge Baary; 13. Arzhan; 14. Suchanicha; 15. Chifeng. 
lítico reciente (Karanovo IV), encontramos en el yacimiento vecino de Kanligecit-Oeste restos de un poblado en una terraza fluvial con cerámicas bien conocidas en Moldavia y el Oeste de Ucrania (Precucuteni). Este hecho es importante, porque demuestra que -posiblemente debido a la llegada de nuevas poblaciones desde el Norte- las estrechísimas conexiones con el interior de Tracia, es decir, con las regiones de la actual Bulgaria que dominan el desarrollo cultural en la zona de Kirklareli durante todo el Neolítico, se cortan completamente. En la primera Edad del Bronce se forma un nuevo 'tell' (Kanligecit-Norte), que supone de nuevo un cambio en las relaciones culturales, si tenemos en cuenta que tanto su arquitectura como su cerámica parecen completamente anatólicas y presentan pocos elementos balcánicos. De especial interés son los tres 'megara', rodeados por una fortificación con entrada de tipo 'propylon', que recuerdan a Troia II, pese a suponérseles una fecha más reciente, en torno al final de la primera Edad del Bronce (Troia V, EB III).

El proyecto germano-ucraniano se concentra en la investigación de la Cultura de Cernoles, es decir, en el final de la Edad del Bronce y su transición a la primera Edad del Hierro en las estepas forestales de Ucrania. Hasta el momento se han realizado campañas de excavaciones en Subotiv, uno de los yacimientos fortificados más importantes de esta cultura, del que se conocen depósitos con bronces de tipo cimerio. Si bien hasta ahora se venía manteniendo la teoría de que las invasiones cimerias en las estepas pónticas acababan con la Edad del Bronce con la consiguiente destrucción de sus poblados y 'ciudades', las nuevas excavaciones han demostrado que algunos de los bronces de tipo cimerio fueron fabricados en las casas del yacimiento de Subotiv (se encontraron incluso moldes para la producción de estos tipos). Este ejemplo es suficiente para demostrar que no solamente la datación de estos llamados bronces cimerios, sino también su valoración histórica, necesitan una reinvestigación profunda en la que nuestras excavaciones en Subotiv, sin lugar a dudas, pueden aportar importantes y definitivos datos.

Cerca de la desembocadura del río Don en el Mar de Azov se encuentra la colonia griega de Tanais, en la Rusia Meridional. Desde las excavaciones rusas de 1955 hasta ahora se han abierto amplios espacios de investigación. A pesar de ello, ha quedado en la oscuridad su historia más antigua. Por esta causa la expedición germano- rusa ha tratado de concentrarse en la apenas conocida época helenística de Tanais (Arsen'eva y Böttger, 1996; Böttger, 1995). Ya en las primeras campañas de excavaciones se encontró el 'agora' del siglo III a.C. con 'propylon', otros restos de arquitectura monumental helenística e inscripciones griegas. Actualmente se está investigando en otra zona de su perímetro un barrio de los siglos IV y V d.C., es decir del Bajo Imperio y la época de las Migraciones. Gracias a los resultados obtenidos se puede hacer una nueva valoración del final de la historia de Tanais, sobre todo a partir de las grandes cantidades de materiales germánicos recogidos (ostrogodos, cultura de Cherniachov), hasta ahora desconocidos en el lugar.

En el Caúcaso la 'Eurasien-Abteilung' mantiene dos proyectos. Excavaciones germano-georgianas investigan varios sitios pre- y protohistóricos en la llanura de Siraki, una región en el extremo sureste de Georgia de especial interés porque se encuentra justo en la frontera con Azerbaiyan. Hasta allí llegaron los antiguos caminos comerciales y militares desde Persia hacia el Caúcaso. De ahí la importancia de la llanura de Siraki para entender las conexiones de las culturas transcaucásicas y el Sur, tal como vemos en varios lugares de esta zona con materiales puramente aqueménidas de los siglos VI a IV a.C. El yacimiento de Ciskaraant-Gora, situado en esta llanura, precede inmediatamente a este horizonte persa. Allí se ha excavado un nivel de destrucción con arquitectura de adobes y gran cantidad de materiales del siglo VII a.C. En este mismo estrato aparecen también numerosas puntas de flecha de tipo escita. No obstante, esperamos encontrar más datos para llevar a cabo con exactitud la interpretación del desarrollo cultural e histórico previo a la instalación de puntos de control persas de época aqueménida en Transcaucasia, una problemática hasta ahora no solamente poca estudiada, sino también apenas conocida en esta región entre los mares Negro y Caspio.

Por otro lado, un equipo germano-dagestano se dedica desde el año 1997 al estudio del 'limes' septentrional del imperio de los Sasánides en Derbent, en la orilla occidental del Mar Caspio. Este se compone de una muralla que incluye varios castillos a lo largo de $40 \mathrm{kms}$ desde el Mar Caspio hacia el Oeste hasta la cima del Caúcaso. Esta muralla es muy importante por su tipo de construcción, conociéndose un único paralelo en el santuario de Takht-i Sulaiman en el Noroeste de Irán, 
antigua excavación también del Instituto Arqueológico Alemán ('Abteilung Teheran'). La datación de este último gira en torno a finales del siglo $\mathrm{V}$ y principios del VI d. C y nuestro actual proyecto intenta la busqueda de una documentación lo más completa posible en este importantísimo monumento arquitectónico, así como la separación de las distintas etapas de construcción y el estudio de las numerosas inscripciones sasánidas en pahlavi.

Existen además varios proyectos en curso en la zona del Asia Central. En Dzharkutan-Tepe en el Sureste de Uzbekistán, a la vez el Noroeste de la antigua Bactria, se encuentra en excavación, junto con el Instituto Arqueológico de la Academia de Uzbekistán en Samarkanda, uno de los yacimientos de la Edad del Bronce más importantes de todo Asia Central. Los trabajos se concentran en la ciudadela, contando hasta el momento con un palacio y un área cultual con torres redondas y un altar en el interior. Esta último tipo de construcción se ha considerado muchas veces como prototipo de los posteriores templos del fuego zoroastrianos. La arquitectura y la cerámica de esta 'ciudad' del Bronce final recuerdan a sitios excavados de la misma época en el Sur de Turkmenistán y en el Norte de Afganistán. Al lado de esta 'ciudad' se encuentra una inmensa necrópolis, en la que las numerosas superposiciones y relaciones estratigráficas de todo tipo entre las tumbas permiten señalar la existencia de varias etapas del Bronce Final en esta zona. Hasta ahora se han excavado más de mil tumbas, pero solamente una pequeña parte está publicada. En el marco de este proyecto se pretende también estudiar y publicar estos inventarios, ya que son una buena parte de la base de la cronología interna del Bronce Final en Bactria.

A unos pocos kilómetros de Dzharkutan se encuentra Dzhandaulat-Tepe, uno de los 'tell' más grandes del Sureste de Uzbekistán. En el siglo VI a.C. Bactria se incorporaba al imperio persa y, tras la llegada de Alejandro Magno a esta región tan alejada del Mundo Antiguo, se formó aquí un imperio grecobactrio con una interesante cultura, mezcla de elementos grecohelenísticos y centralasiáticos. Pero el florecimiento cultural y económico de esta zona se concentró a lo largo del reino de la dinastía de Kushan con su budismo grecoindio hasta que, en época sasánida (siglo III/IV d.C.), Bactria cayó de nuevo bajo el dominio persa. La importancia de Dzhandaulat-Tepe se basa, al parecer, en el hecho de ser uno de los pocos sitios habitados durante todo este tiempo, sin gran- des interrupciones. De ahí que se trate de un lugar 'modélico' para estudiar los cambios culturales entre el siglo VI a.C. y el siglo IV d.C.

Con el fin de investigar la minería y metalurgia del estaño en el Asia central preislámica, ha comenzado un proyecto, financiado por la fundación Volkswagen, en cooperación con el Deutsche Bergbau-Museum en Bochum y el Max-PlankInstitut für Kernphysik en Heidelberg y con las Academias de Uzbekistán y Tachikistán. En la Antigüedad, y posiblemente ya en época prehistórica, el estaño de Asia Central jugaba un papel importantísimo en todo el Oriente. Se sabe también por prospecciones geológicas que Afganistán, Tachikistán, Uzbekistán y -en parte- también Kirgistán y Kazajstán poseen ricos yacimientos de estaño. Pero hasta ahora nunca se habían estudiado los principios de su beneficio y uso en estas regiones. Por ello se comenzó en el año 1997 la investigación arqueológica y geológica de los entornos de los importantes yacimientos de estaño de Karnab, en el valle del río Serafshan entre Samarkanda y Bujara, Uzbekistán, y a 3000 m. de altura en Mushiston (Lám. I), cerca de Pendzhikent en el Noroeste de Tachikistán. Hasta ahora, y por lo menos en Mushiston, hemos podido comprobar que el beneficio del estaño en las minas empezaba ya en la Edad del Bronce, es decir en la primera mitad del segundo milenio a.C. (cerámica de la Cultura de Andronovo).

Otra zona donde se concentra la investigación de la 'Eurasien-Abteilung' son las estepas de Siberia y sus regiones limítrofes. Sobre ello quiero tratar aquí más en detalle. Las primeras expediciones de carácter arqueológico tuvieron lugar en

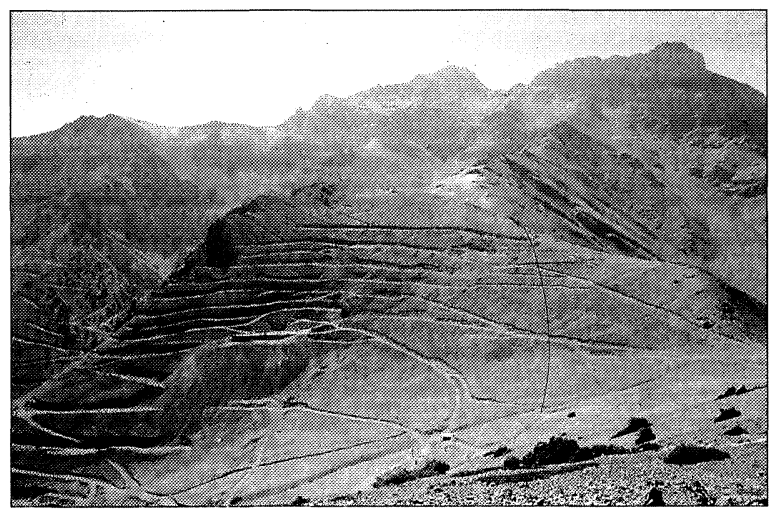

Lám. I. Mushiston cerca de Pendzhikent, en el noroeste de Tachikistán. El yacimiento de estaño con visibles restos de su beneficio en época moderna. 
la época de Pedro el Grande. Su interés por la búsqueda de oro y otros minerales conllevó una serie de expediciones a las, en aquel tiempo, casi desconocidas regiones de Siberia. Muchas de ellas fueron dirigidas por científicos alemanes del siglo XVIII, al servicio de los Zares de Rusia. Una de éstas tuvo lugar en el año 1720, dirigida por D.G. Messerschmidt, quien excavó un 'kurgán' a las orillas del río Yenisei por primera vez con métodos científicos. Pocos años despues G.F. Müller y J.G. Gmelin se planteaban -también por primera vez- una serie de cuestiones de carácter cronológico sobre los hallazgos de Siberia. En 1863 el alemán W. Radloff al servicio del Zar, dirigía otra expedición en la llanura de Minusinsk, zona de Siberia donde se encuentran numerosas estelas con inscripciones rúnicas. Aunque en el siglo pasado se consideraban estas estelas como restos de los proto-finougrios, Radloff fue el primero que pudo comprobar que en realidad se trataban de inscripciones de los antiguos pueblos de lengua turca en esta región en los siglos V, VI y VII d.C. (Kossack, 1995). Todas estas investigaciones, sin embargo, han dejado claro que las estepas alrededor del Yenisei, en especial la llanura de Minusinsk, son una de las zonas arqueológicamente más ricas y más interesantes de Eurasia (Tallgren, 1925).

Otro destino llevaba a G. v. Merhart al Yenisei. A comienzos de la Primera Guerra Mundial fué enviado al frente en Galizia y, ya en el primer año de 1914, hecho prisonero. Después de varios años en distintos campos en Siberia, en el 1919 fue enviado al Museo de la Sociedad Geográfica de Krasnoiarsk. Allí ordenó la colección arqueológica y llevó a cabo -junto con científicos rusos como S. Teplouchov- pequeñas excavaciones en el Yenisei. Cuando en el año 1920 empezaron a repatriar a los prisioneros de guerra alemanes, Merhart se quedó allí voluntariamente un año más para terminar el trabajo empezado. El resultado científico de esta estancia fue su libro La Edad del Bronce en el Yenisei, publicado en el año 1926 (Merhart, 1926).

En los siguientes setenta años el contacto científico no fue nunca demasiado estrecho, resultando impensable llevar a cabo excavaciones comunes en Siberia o en cualquier otra parte de la antigua URSS, hasta que los cambios políticos de principios de los años noventa crearon un clima distinto, en el cual se pudieron compartir de nuevo una serie de proyectos científicos con nuestros co- legas rusos. Uno de los problemas más importantes que se plantean en la actualidad es el desarrollo cultural desde la primera Edad del Bronce hasta la Cultura de Tagar de época escita (Vadeckaja, 1986; Heidenreich, 1990); es decir, la génesis de los rituales funerarios, del estilo animalístico y de otros elementos de la Cultura de Tagar desde sus raíces más antiguas y el papel que en este desarrollo tuvieron las influencias meridionales, desde Mongolia y el Norte de China.

La 'Eurasien-Abteilung' desde el año 1995 está llevando a cabo, en cooperación con el Museo de Minusinsk, excavaciones en una serie de necrópolis situadas en una de las terraza altas del Yenisei, bajo el monte Suchanicha. Las investigaciones incluyen también el cementerio más pequeño de Potrosilovo, a pocos kilómetros de Suchanicha. La necrópolis de Suchanicha (Lám. II) es sumamente interesante, no solamente porque se encuentra en una zona central dentro de la llanura de Minusinsk, donde el río Tuba desemboca en elYenisei, sino también y sobre todo, porque en este cementerio están representadas prácticamente todas las culturas pre- y protohistóricas del Sur de Siberia. De ahí que este lugar desde el principio se considerase ideal para la solución de enigmas cronológicos y relativos al desarrollo cultural. Las primeras campañas de excavaciones confirmaron esta idea: en Suchanicha y Potrosilovo se excavaron numerosas tumbas de las Culturas Afanas'evo, Okunev, Andronovo, Karasuk und Kamennyj Log, es decir del Eneolítico hasta el Bronce Final, además de otras de los Hunos y de la Cultura de Tashtyk, quedando por investigar para los próximos años los típicos 'kurganes' con estelas de la cultura de Tagar de época escita.

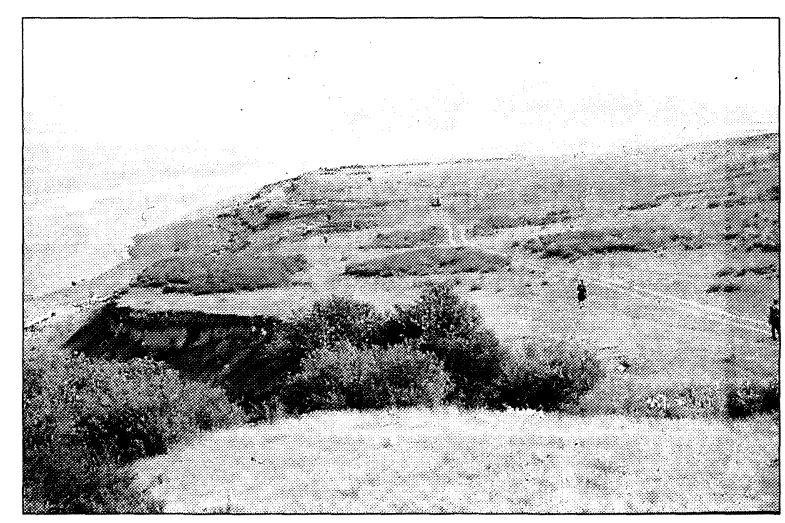

Lám. II. Suchanicha, en el sur de Siberia. Vista de la necrópolis en la orilla alta del Yenisei. 
En las excavaciones en Suchanicha se recogieron también restos orgánicos de varios culturas para su datacion por C14 en el laboratorio de la 'Eurasien-Abteilung' en Berlin. Después de contar con los primeros resultados en este campo, podemos afirmar una antigüedad superior en 500 años a las fechas que se sostenían anteriormente para casi todas las culturas del Sur de Siberia. Por ejemplo, la Cultura eneolítica de Afanas'evo tendrá que ser transvasada desde la segunda mitad del tercer milenio a.C. a la primera mitad de este mismo milenio y hasta finales del cuarto milenio a.C. De especial interés es también la datación de la fase Kamennyj Log: una etapa de transición de la Cultura de Karasuk del Bronce Final a la de Tagar de época escita, fechada hasta ahora en el siglo IX/ VIII a.C. Nuestras fechas de Suchanicha para Kamennyj Log, sin embargo, se concentran en el último tercio del segundo milenio a.C. (Leont'ev et alii, 1996). Ello significa que también los principios de la cultura de Tagar y del estilo animalístico tienen una datación más antigua, resultado que se corresponde ahora mucho mejor con dataciones históricas y de C14 de hallazgos en el Norte de China, sincronizados con Karasuk y Kamennyj Log (fase de Anyang, dinastía tardía Shang).

Las investigaciones de la 'Eurasien-Abteilung' en el Sur de Siberia no se deben concentrar exclusivamente en la llanura de Minusinsk sino que, para entender mejor las influencias del Sur en este proceso de desarrollo cultural, hay que incluir en el proyecto lugares al sur de las montañas Sayan, importantes caminos antiguos que, partiendo del Norte de China y cruzando Mongolia, llegan a Tuva en el alto Yenisei y desde allí -cruzando el Sayan occidental-al Norte de la región de Minusinsk. Justamente al sur del Sayan occidental, a ambos lados del río Uyuk, se encuentra la llanura de Arzhan (Lám. III), donde existe una de las necrópolis más impresionantes de la época escita en Eurasia. M.P. Grjaznov (1984) investigó aquí en los años setenta un 'kurgan' con más de 100 m. de diámetro y con una construcción de madera en el interior, que todavía hoy sigue siendo completamente singular. El inventario de sus materiales ayudó a definir un horizonte muy antiguo de la cultura escita.

Es característico de la llanura de Arzhan que los 'kurganes' formen líneas de muchos kilómetros a través de toda la llanura. Por ello la 'Eurasien-Abteilung', junto con arqueólogos de St. Petersburgo, ha comenzado la excavación de uno de

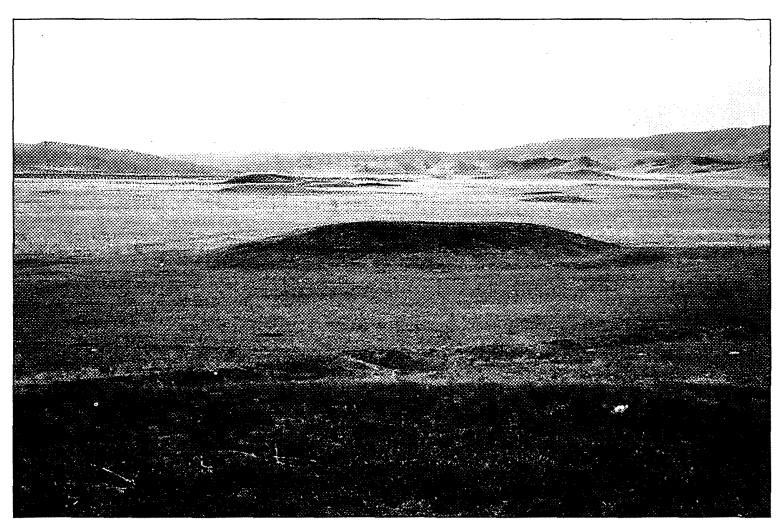

Lám. III. Arzhan, Tuva. Llanura con cadenas de 'kurganes' escitas.

estos 'kurganes' en una de las cadenas al oeste del actual pueblo de Arzhan (Lám. IV). Bajo un túmulo de tierra de $30 \mathrm{~m}$. de diámetro y tapada por una capa de piedras, se encontraba la cámara funeraria, construída con troncos de alerce. El ajuar, sobre todo unos pequeños pájaros de chapa de oro, se fecha en el siglo $\mathrm{V}$ a.C., es decir en la fase de Pazyryk, la época de florecimiento de la cultura de los Escitas. Una prospección en los alrededores nos llevó a pensar que posiblemente al oeste de Arzhan, donde se excavaba en 1997, se encuentren los 'kurganes' más tardíos, mientras al este del pueblo se hallan plataformas de piedras bastante más antiguas, una de las cuales fue excavada por Grjaznov. Esta hipótesis nos llevará a intentar comprobar en los próximos años la posible existencia de algún tipo de estratigrafía horizontal en la llanura de Arzhan.

Más hacia el Sur, la estepa cambia hacia un tipo de semidesierto. En Tuva central empieza un co-

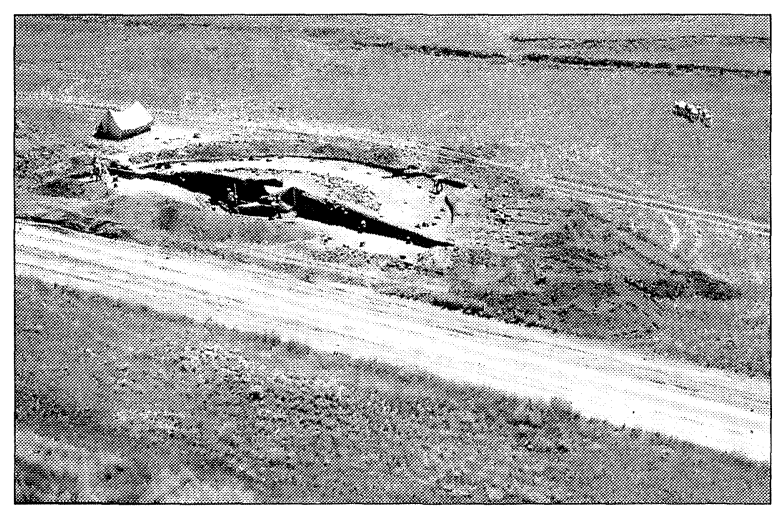

Lám. IV. Arzhan, Tuva. Excavaciones de la EurasienAbteilung en un 'kurgan' escita en 1997. 
rredor que se abre hacia Mongolia, y a través del cual corre también hoy en día la actual carretera entre Kyzyl, la capital de Tuva, y el Noroeste de Mongolia. Cerca de Kyzyl se encuentra otra necrópolis escita en Doge Baary, donde -como en Arzhan- los 'kurganes' forman cadenas. Arqueólogos de St. Petersburgo trabajaban ya varios años en este cementerio cuando en 1996 la 'EurasienAbteilung' entró a formar parte de este proyecto, que contaba ya con importantes resultados en comparación con Arzhan.

Para entender mejor el desarrollo cultural en el sur de Siberia y su relación con las otras regiónes de la estepa euroasiática es importante no solamente seguir el camino hacia el Sur y Sureste, sino también hacia el Oeste. Son de sobra conocidas influencias artísticas como el estilo animalístico que, debido a una serie de migraciones desde el interior de Asia, llegaban hasta la zona del Mar Negro e incluso más hacia el Oeste. Por ello era necesario investigar las distintas etapas de este camino hacia el Oeste y, de acuerdo con este planteamiento, se comenzó en el año 1997, en colaboración con la Universidad de Petropavlovsk, un proyecto en las estepas en el norte de Kazajstan, en especial en la necrópolis de Bajkara cerca de Sergeevka (Lám. V).

En la primera campaña se descubrieron tumbas de la Edad del Bronce y de los antiguos pueblos de lengua turca, lo que hace pensar que también este cementerio fue usado como el de Suchanicha durante muchos siglos. Sin embargo, el centro de la necrópolis lo forma un 'kurgan' monumental de $82 \mathrm{~m}$. de diámetro y $7 \mathrm{~m}$. de altura. Una de sus esquinas estaba destrozada y allí se procedió en

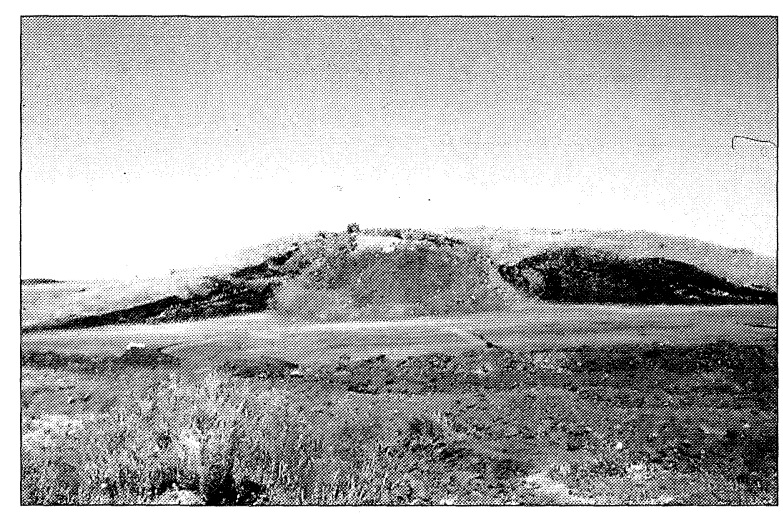

Lám. V. Bajkara cerca de Sergeevka, en el norte de Kazajstan. Excavaciones de la Eurasien-Abteilung en un 'kurgan' escita en 1997.
1997 a la limpieza del perfil, tras la cual pudimos comprobar que el monumento contaba al menos con cuatro 'kurganes' distintos, uno encima del otro. En consecuencia los próximos años se dedicarán a su excavación sistemática, en busca de los datos necesarios para la elaboración, mediante una estratigrafía vertical, de una cronología de la primera Edad del Hierro.

Las excavaciones en Suchanicha, Potrosilovo, Arzhan, Doga Baary y Sergeevka se completan con otros estudios. Por ejemplo, en 1996 llevamos a cabo en colaboración con arqueólogos de St. Petersburgo una prospección enTuva occidental, una región de especial interés situada entre Rusia, Kazajstan, China y Mongolia, y apenas conocida arqueológicamente. El acceso a los valles y montañas de estas regiones es sumamente difícil.Todavía hoy la población tuvina vive allí de la transhumancia: durante el invierno se asientan en las llanuras bajas de los ríos, y cuando empieza el verano migran con sus rebaños a través de los valles hacia las montañas. A lo largo de estos caminos hemos podido documentar la existencia de numerosos cementerios, fechables desde la Edad del Bronce hasta época medieval e, incluso, hasta nuestros días.

Otro de nuestros proyectos se dedica a la publicación y análisis de los más de 9.000 bronces pertenecientes a la Cultura de Tagar y depositados en el Museo de Minusinsk (Lám. VI), que contiene a su vez una de las colecciones arqueológicas más ricas de Siberia. Como muchos de estos bronces están decorados en el estilo animalístico, es de esperar contar con nuevos resultados en relación con el origen y desarrollo de este arte tan típico de las estepas de Eurasia.

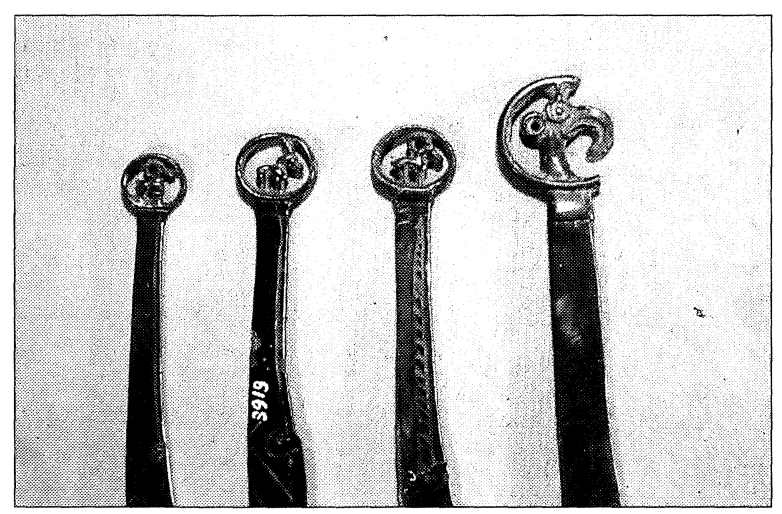

Lám. VI. Bronces decorados en el estilo animalístico de la Cultura de Tagar, Museo de Minusinsk. 
Finalmente, para un mejor entendimiento de las influencias desde el Sur, es indispensable poder incluir en nuestros intentos el norte de China. Por esta razón las colaboraciones con los Institutos Arqueológicos en Pekin y en Hohot, provincia de Mongolia Interior, incluyen publicaciones conjuntas de materiales importantes para las culturas de Eurasia, investigaciones en el campo de las dataciones de $\mathrm{C} 14$ y de la dendrocronología y -en el futuro- también excavaciones. Además en el borde meridional de las estepas, se encuentran una serie de 'tells' que ofrecen posibilidades ideales para la solución de problemas cronológicos y culturales. De momento, los materiales recogidos en superficie confirman tanto las conexiones con el Norte como con las culturas de China Central en el Sur.

En esta breve presentación de las actividades de la 'Eurasien-Abteilung' del InstitutoArqueológico Alemán en las estepas de Eurasia, hemos mostrado algunos ejemplos de las cuestiones que mueven nuestra investigación, así como de sus primeros resultados. Resumiendo, podemos afirmar que nuestros proyectos se concentran en aquellas regiones como el Mar Negro, el Caúcaso, Asia Central y Siberia, donde las civilizaciones del Sur-Grecia, Persia, el valle del Indo o Chinainfluyeron fuertemente en el proceso de formación cultural. Todos nuestros proyectos son recientes, algunos no han comenzado antes de 1997; pese a ello se encuentran en buen camino. Como se puede entender el trabajo en estos países no es fácil y las dificultades logísticas son algunas veces extremas. No obstante podemos decir con satisfacción que la colaboración científica con los distintos Institutos de las Academias, con las Universidades y con los Museos se desarrollan bien y nos permiten mirar con optimismo hacia el futuro.

\section{BIBLIOGRAFÍA (1)}

Arsen'eva, T. y Böttger, B. (1996): “Griechen am Don. Die Grabungen in Tanais 1995". Eurasia Antiqua, 2: 405-453.

BötTger, B. (1995): "Griechen am Don. Die Ausgrabungen in Tanais". Archäologischer Anzeiger: 99-118 .

GrJaznov, M.P. (1984): Der Großkurgan von Arzan in Tuva, Südsibirien. Materialien zur Allgemeinen und Vergleichenden Archäologie, 23. München.

HeIDENREICH, H. (1990): Die sibirische Tagar-Kultur. Ein Forschungsbericht. Kleine Schriften aus dem Vorgeschichtlichen Seminar der Philipps-Universität Marburg, 32. Marburg.

Kossack, G. (1995): "Geschichte und Aufgaben der archäologischen Erforschung Mittelasiens an der Schwelle zur frühen Eisenzeit". Eurasia Antiqua, 1: 17-43.

Leont'ev, N.; Parzinger, H.y Nagler, A. (1996): “Die russisch-deutschen Ausgrabungen beim Berg Suchanicha am mittleren Enisej”. Eurasia Antiqua, 2: 175204.

Merhart, G. von (1926): Bronzezeit am Jenissei. Ein Beitrag zur Urgeschichte Sibiriens. Wien.

PARZINGER, H. (1995): "Perspektiven und Forschungsvorhaben der Eurasien-Abteilung des Deutschen Archäologischen Instituts". Eurasia Antiqua, 1: 7-14.

Tallgren, A. (1925): "Eurasische Bronzezeit". En M. Ebert (ed.): Reallexikon der Vorgeschichte, 3. Berlin: 151-152.

VADECKAJA, E.B. (1986): Archeologičeskie pamjatniki v stepjach Srednego Eniseja. Leningrad.

(1) Seleccionada

T. P., 55, n. $^{\circ} 1,1998$ 